\title{
Dietary inflammatory index and anthropometric measures of obesity in a population sample at high cardiovascular risk from the PREDIMED (PREvención con Dleta MEDiterránea) trial
}

M. Ruiz-Canela ${ }^{1,2 *}$ I. Zazpe ${ }^{2,3}$, N. Shivappa ${ }^{4,5}$, J. R. Hébert ${ }^{4,5}$, A. Sánchez-Tainta ${ }^{2}$, D. Corella ${ }^{2,6}$, J. Salas-Salvadó ${ }^{2,7}$, M. Fitó ${ }^{2,8}$, R. M. Lamuela-Raventós ${ }^{2,9}$, J. Rekondo ${ }^{2,10}$, J. Fernández-Crehuet ${ }^{2,11}$, M. Fiol ${ }^{2,12}$, J. M. Santos-Lozano ${ }^{2,13}$, L. Serra-Majem ${ }^{2,14}$, X. Pinto ${ }^{2,15}$, J. A. Martínez ${ }^{2,3}$, E. Ros ${ }^{2,16}$, R. Estruch ${ }^{2,17}$ and M. A. Martínez-González $z^{1,2}$

${ }^{1}$ Department of Preventive Medicine and Public Health, Facultad de Medicina-Clinica Universidad de Navarra, School of Medicine, Universidad de Navarra, Irunlarrea 1, 31080 Pamplona, Navarra, Spain

${ }^{2}$ CIBER Fisiopatología de la Obesidad y Nutrición (CIBER obn), Instituto de Salud Carlos III, Madrid, Spain

${ }^{3}$ Department of Nutrition and Food Sciences, University of Navarra, Pamplona, Spain

${ }^{4}$ Cancer Prevention and Control Program, University of South Carolina, Columbia, SC, USA

${ }^{5}$ Department of Epidemiology and Biostatistics, Arnold School of Public Health, University of South Carolina, Columbia, SC, USA

${ }^{6}$ Department of Preventive Medicine, University of Valencia, Valencia, Spain

${ }^{7}$ Human Nutrition Unit, Sant Joan University Hospital, IISPV, Universitat Rovira i Virgili, Reus, Spain

${ }^{8}$ Inflammatory and Cardiovascular Disease Programme (RICAD), IMIM-Hospital del Mar Medical Research Institute,

Barcelona, Spain

${ }^{9}$ Department of Nutrition and Food Science, School of Pharmacy, Instituto de Investigación en Nutrición y Seguridad Alimentaria, University of Barcelona, Barcelona, Spain

${ }^{10}$ Department of Cardiology, University Hospital of Alava, Vitoria, Spain

${ }^{11}$ Department of Preventive Medicine, University of Malaga, Malaga, Spain

${ }^{12}$ Institute of Health Sciences (IUNICS), University of Balearic Islands, Palma de Mallorca, Spain

${ }^{13}$ Department of Family Medicine, San Pablo Health Center, Primary Care Division of Sevilla, Andalusian Health Service \& Department of Medicine, University of Sevilla, Spain

${ }^{14}$ Research Institute of Biomedical and Health Sciences, University of Las Palmas de Gran Canaria, Las Palmas de Gran Canaria, Spain

${ }^{15}$ Lipids and Vascular Risk Unit, Internal Medicine, Hospital Universitario de Bellvitge, Hospitalet de Llobregat, Barcelona, Spain

${ }^{16}$ Lipid Clinic, Department of Endocrinology and Nutrition, Institut d'Investigacions Biomèdiques August Pi Sunyer

(IDIBAPS), Hospital Clinic, Barcelona, Spain

${ }^{17}$ Department of Internal Medicine, IDIBAPS, Hospital Clinic, University of Barcelona, Barcelona, Spain

(Submitted 26 July 2014 - Final revision received 3 December 2014 - Accepted 5 December 2014 - First published online 27 February 2015)

\section{Abstract}

The dietary inflammatory index (DII) is a new tool to assess the inflammatory potential of the diet. In the present study, we aimed to determine the association between the DII and BMI, waist circumference and waist:height ratio (WHtR). We conducted a cross-sectional study of 7236 participants recruited into the PREvención con DIeta MEDiterránea trial. Information from a validated 137-item FFQ was used to calculate energy, food and nutrient intakes. A fourteen-item dietary screener was used to assess adherence to the Mediterranean diet (MeDiet). Sex-specific multivariable linear regression models were fitted to estimate differences (and 95\% CI) in BMI, waist circumference and WHtR across the quintiles of the DII. All nutrient intakes, healthy foods and adherence to the MeDiet were higher in the quintile with the lowest DII score (more anti-inflammatory values) except for intakes of animal protein, saturated fat and monounsaturated fat. Although an inverse association between the DII and total energy was apparent, the DII was associated with higher average BMI, waist circumference and WHtR after adjusting for known risk factors. The adjusted difference in the WHtR for women and men between the highest and

Abbreviations: CRP, C-reactive protein; DII, dietary inflammatory index; MeDiet, Mediterranean diet; PREDIMED, PREvención con DIeta MEDiterránea; WHtR, waist:height ratio.

*Corresponding author: M. Ruiz-Canela, fax: +34948 4256 49, email mcanela@unav.es 
lowest quintiles of the DII was $1.60 \%(95 \%$ CI 0.87, 2.33) and 1.04\% (95\% CI 0.35, 1.74), respectively. Pro-inflammatory scores remained associated with obesity after controlling for the effect that adherence to a MeDiet had on inflammation. In conclusion, the present study shows a direct association between the DII and indices of obesity, and supports the hypothesis that diet may have a role in the development of obesity through inflammatory modulation mechanisms.

\section{Key words: Inflammation: Diet: Obesity: BMI: Waist circumference: Waist:height ratio}

The obesity pandemic constitutes a major public health problem in most high-income countries, and it is emerging as a threat in more affluent sectors of developing countries ${ }^{(1)}$. In 2008 , more than $10 \%$ of the World's adult population, i.e. about 500 million people, were obese according to the $\mathrm{WHO}^{(2)}$. It was estimated that 3.4 million adult deaths worldwide were, in 2010 , attributable to obesity or overweight ${ }^{(1)}$. This is a global crisis because $65 \%$ of the world's population live in countries where overweight and obesity kill more people than being underweight ${ }^{(2)}$.

Obesity usually is the result of the accumulation of excess body fat, and it is often characterised as a state of low-grade chronic inflammation ${ }^{(3)}$. This obesity-induced inflammation has multi-organ metabolic effects affecting the adipose tissue, liver, muscle, pancreas and brain ${ }^{(4)}$. Metabolic differences exist according to the location of fat cells. For example, excessive deposition of fat in visceral adipose tissue (i.e. intra-abdominal fat) is associated with higher health risks than subcutaneous fat accumulation in the extremities ${ }^{(5)}$. In fact, different anthropometric adiposity measures including waist circumference or waist:height ratio (WHtR) are used to assess the role of adiposity in CVD risk $^{(6,7)}$.

A number of studies have shown an association between diet and inflammatory biomarkers, and how this translates into increased or decreased risk of chronic metabolic diseases $^{(8-15)}$. Part of the preventive role of healthy dietary patterns, such as the Mediterranean diet (MeDiet), could be attributed to the anti-inflammatory properties of some of their main components ${ }^{(15-19)}$. This anti-inflammatory effect may decrease the low-grade inflammation usually found in obese patients $^{(20,21)}$. However, a MeDiet may also attenuate inflammation in the absence of weight loss ${ }^{(22)}$. A recent hypothesis is that obesity could also be partly the consequence of a previous chronic low-grade inflammation; therefore, a bidirectional association between inflammation and obesity may exist ${ }^{(23)}$

Consequently, it can be useful to characterise an individual's diet according to its inflammatory properties in order to investigate the inflammatory links between obesity and diet ${ }^{(24)}$. The dietary inflammatory index (DII) is a new tool to assess this inflammatory potential of the diet ${ }^{(25)}$. In the present study, we examine the relationships between nutrient intake or food group consumption and the DII, as well as the association between the DII and indices of both general and abdominal obesity in the PREvención con DIeta MEDiterránea (PREDIMED) trial.

\section{Methods}

\section{Ethics statement}

The protocol was approved by the Research Ethics Committees at all recruiting centres: University of Navarra; University of Valencia; University Rovira i Virgili; IMIM-Hospital del Mar Medical Research Institute; University of Barcelona; University Hospital of Alava; University of Malaga; University of the Balearic Islands; University of Las Palmas de Gran Canaria; University Hospital of Bellvitge; Hospital Clinic. Participants signed a written informed consent form.

\section{Study design and participants}

The 'PREDIMED' study was a parallel-group, multi-centre, clinical trial that aimed to assess the effects of the traditional MeDiet on the primary prevention of CVD (protocol available at http://www.predimed.es). A detailed description of methods and patients has been published elsewhere ${ }^{(26,27)}$. The study was conducted between October 2003 and December 2010 by eleven recruiting centres in Spain.

Eligible participants were men aged 55-80 years and women aged 60-80 years with no previous CVD. At baseline, participants should have a diagnosis of type 2 diabetes mellitus or at least three of the following major cardiovascular risk factors: smoking (more than one cigarette per d during the last month); hypertension (systolic blood pressure $\geq 140 \mathrm{mmHg}$ or diastolic blood pressure $\geq 90 \mathrm{mmHg}$ or antihypertensive medication); elevated LDL-cholesterol levels ( $\geq 1600 \mathrm{mg} / \mathrm{l}$ ); low HDL-cholesterol levels $(\leq 400 \mathrm{mg} / \mathrm{l}$ in men or $\leq 500 \mathrm{mg} / \mathrm{l}$ in women, independently of lipid-lowering therapy); BMI $\geq 25 \mathrm{~kg} / \mathrm{m}^{2}$; family history of premature CHD.

A total of 7447 participants were randomised in a 1:1:1 ratio to a parallel-design intervention trial of dietary advice: (1) a MeDiet supplemented with extra-virgin olive oil; (2) a MeDiet supplemented with nuts; (3) a low-fat diet (control group). Medical conditions and risk factors related to eligibility were collected using a questionnaire during the first screening visit. Participants, with the assistance of trained dietitians, completed an FFQ. This FFQ was adapted from the Willett questionnaire and validated in Spain ${ }^{(28)}$. It includes 137 items plus vitamin/mineral supplements, and specific questions for patterns of alcohol consumption. Energy and nutrient intakes were calculated from Spanish food composition tables ${ }^{(29)}$. Participants also completed the Spanish validated version of the Minnesota physical activity questionnaire ${ }^{(30)}$, and a fourteen-item dietary screener to assess the adherence to the MeDiet ${ }^{(31)}$. PREDIMED dietitians were responsible for the accurate completion of the questionnaires.

For the present study, 133 participants were excluded from the analyses because they reported values for total energy intake outside of the predefined limits $(<3347 \mathrm{~kJ}(<800 \mathrm{kcal}) / \mathrm{d}$ or $>17573 \mathrm{~kJ}(>4200 \mathrm{kcal}) / \mathrm{d}$ for men; $<2510 \mathrm{~kJ}(<600 \mathrm{kcal}) / \mathrm{d}$ or $>14644 \mathrm{~kJ}$ ( $>3500 \mathrm{kcal}) / \mathrm{d}$ for women). These limits were set in accordance with those recommended by Willett in Nutritional Epidemiology ${ }^{(32)}$. Another seventy-eight participants were excluded 
because of lack of information on the FFQ needed to calculate the DII. This study was registered as an International Standard Randomised Controlled Trial, number ISRCTN35739639.

\section{Dietary inflammatory index}

The design and development of the DII has been described elsewhere $^{(25)}$. Briefly, the DII is a scoring algorithm based on an extensive review of the literature published from 1950 to 2010 , linking 1943 articles to a total of forty-five food parameters and including various macronutrients, micronutrients, flavonoids and food items (Fig. 1). These dietary parameters were scored according to whether they increased $(+1)$, decreased $(-1)$ or had no effect $(0)$ on six inflammatory biomarkers (IL-1 $\beta$, IL-4, IL-6, IL-10, TNF- $\alpha$ and C-reactive protein (CRP)). An overall food parameter-specific inflammatory effect score was calculated and multiplied by a centred percentile value for each food. This percentile was calculated by first linking the dietary data from a study to the regionally representative world database intake, which was based on actual human consumption in eleven populations from different parts of the world that provided a robust estimate of a mean and standard deviation for each parameter. These values then become the multipliers to express an individual's exposure, relative to the 'standard global mean' as a $z$-score. This was achieved by subtracting the 'standard global mean' from the amount reported, and dividing this value by the standard deviation. To minimise the effect of 'right skewing', this value was then converted to a centred percentile score. The centred percentile score for each food parameter for each individual was multiplied by the respective food parameter effect score that was derived from the literature review in order to obtain a food parameter-specific DII score for an individual. All of the food parameter-specific DII scores were then summed to create the overall DII score for every participant in the study. The greater the DII score, the more pro-inflammatory the diet, and more negative values represent more anti-inflammatory diets. The DII score could take on values ranging from 7.98 (maximally pro-inflammatory) to -8.87 (maximally anti-inflammatory) ${ }^{(25)}$.

Construct validation of the DII was performed using data derived from two different sources of dietary intake information, and serum high-sensitivity CRP as the construct validator $^{(33)}$.

\section{Outcome}

Trained and certified PREDIMED nurses performed all baseline anthropometric adiposity measures including weight and height (from which BMI $\left(\mathrm{kg} / \mathrm{m}^{2}\right)$ was computed), waist circumference $(\mathrm{cm})$ and WHtR $(\%)$ following validated procedures. A waist:height ratio equal to 1 was taken as $100 \%$. Baseline weight was measured using a calibrated balance beam scale with the subjects barefoot and wearing light clothes. The nurse measured height using a wall-mounted calibrated stadiometer. Waist circumference was measured using an anthropometric measuring tape, at a horizontal plane midway between the lowest rib and the iliac crest.

\section{Statistical analyses}

Statistical analyses were stratified by sex. Comparisons of quantitative variables across the quintiles of the DII were made using a one-way ANOVA. The compared variables included total energy intake, physical activity and nutrient and food consumption. Intakes of carbohydrate, protein and fat (and fat subtypes) are expressed as a percentage of total energy intake (Table 1). Categorical variables were compared using the Pearson $\chi^{2}$ test.

Sex-specific least-squared means of BMI, waist circumference and WHtR were estimated across the quintiles of the DII. Pearson's correlation coefficients (95\% CI) between these anthropometric adipose measures and the DII were also calculated.

Sex-specific multiple linear regression models were used to estimate the differences (and 95\% CI) in the indices of general obesity and abdominal obesity according to the quintiles of the DII. Covariates included in these models were age (years), smoking status (never, current or former smoker), diabetes (yes or no), hypertension (yes or no), leisure-time physical activity (metabolic equivalents-min/d), educational level (illiterate/elementary education, secondary education or university), marital status (married, widowed, single or other), total energy intake $(\mathrm{kJ} / \mathrm{d})$ and study centre. In addition, tests of linear trend across the successive quintiles of the DII were conducted using the median value for each quintile category as a continuous variable, and after adjusting for the aforementioned confounding variables.

Residuals of the DII were obtained in a linear regression analysis of the association between the DII and a previously validated fourteen-item PREDIMED screener of adherence to the MeDiet ${ }^{(31)}$. These residuals represent the information provided by the DII, which is not explained at all by adherence to the MeDiet (i.e. they exhibit zero correlation with the MeDiet score). They were included as an independent variable after transformation into quintiles in a multivariable regression model with the same covariates listed previously (residual model).

All $P$ values presented are two-tailed, and differences were considered statistically significant at $P \leq 0 \cdot 05$. All statistical analyses were performed using STATA ${ }^{\circledR}$ version 12.0 (Stata Corp).

\section{Results}

Of the 7447 initially randomised subjects in the PREDIMED trial, 7236 were included in the present study. The remaining participants ( $n$ 211, 2.8\%) were excluded because of incomplete data on their FFQ ( $n$ 78) or baseline energy intake outside of the predefined values ( $n$ 133). Among the 7236 participants, $57 \%$ were women. The mean age of the participants was 68 (SD 5.8) years for women and 66 (SD 6.6) years for men. The median DII score for women was $-0 \cdot 78$ $(-4.90$ to 3.68$)$ and -0.91 ( -5.23 to 3.69$)$ for men.

Table 1 shows the main characteristics of the participants according to the categories of the DII score by sex. All differences between the quintiles of this index were statistically 
Review of articles published from 1950 to 2010, resulting in 1943 studies linking a total of forty-five food parameters with inflammatory biomarkers

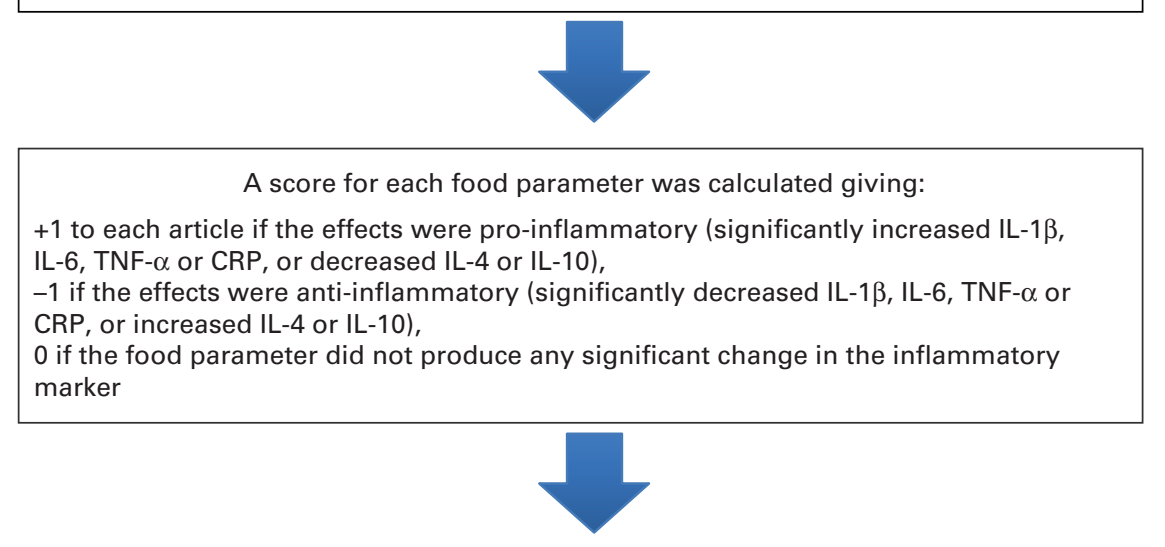

The score for each food parameter was weighted according to the study design. The weights were 10 (experimental design), 8 (observational), 7 (case-control), 6 (cross-sectional), 5 (experimental with animals), 3 (cell culture)

A food parameter-specific overall inflammatory effect score was calculated by substracting the anti-inflammatory fraction from the pro-inflammatory fraction.

This score was corrected if the total weighted number of articles was $<236$. In these cases the raw overall inflammatory score is multiplied by the total weighted number of articles divided by 236

$z$-Score and centred percentiles for each of the thirty-three food parameters* for each participant of the PREDIMED trial were calculated based on the average and standard deviation for each food parameter obtained from the global database that was created from the consumption of the original forty-five food parameters fron eleven countries from around the world

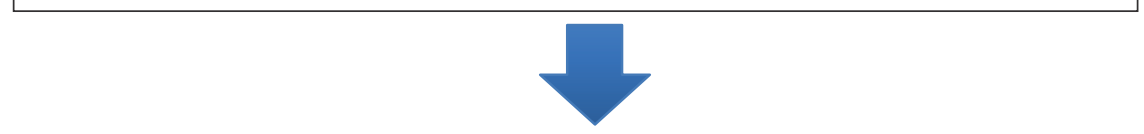

The centred percentile for each food parameter is multiplied by the respective 'overall food parameter-specific inflammatory effect score' to obtain the 'food parameter-specific DIl score'

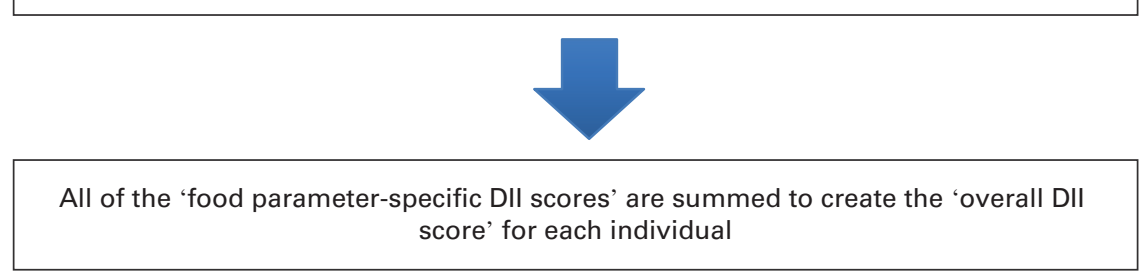

Fig. 1. Sequence of steps in creating the dietary inflammatory index in the PREvención con Dleta MEDiterránea (PREDIMED) trial. *Alcohol, $\beta$-carotene, caffeine, carbohydrate, cholesterol, energy, iron, fibre, folic acid, garlic, green/black tea, magnesium, MUFA, $n-3$ fatty acids, $n-6$ fatty acids, niacin, onion, pepper, protein, PUFA, riboflavin, saturated fat, Se, thiamin, total fat, trans-fat, vitamin A, vitamin $B_{12}$, vitamin $B_{6}$, vitamin $C$, vitamin D, vitamin $E$ and Zn. CRP, C-reactive protein; DII, dietary inflammatory index. (A colour version of this figure can be found online at http://www.journals.cambridge.org/bjn). 
Table 1. Description of the main characteristics of the participants according to quintiles (Q) of the dietary inflammatory index (DII) score in the PREvención con Dleta MEDiterránea (PREDIMED) Trial, 2003-9

(Mean values and standard deviations; median values, minimum and maximum values, and percentages)

\begin{tabular}{|c|c|c|c|c|c|c|c|c|c|c|c|c|}
\hline & \multicolumn{12}{|c|}{ Quintiles of the DII } \\
\hline & \multicolumn{6}{|c|}{ Women } & \multicolumn{6}{|c|}{ Men } \\
\hline & $\mathrm{Q1}^{*}$ & Q2 & Q3 & Q4 & Q5† & $P$ & $\mathrm{Q1}^{*}$ & Q2 & Q3 & Q4 & Q5† & $P$ \\
\hline \multicolumn{13}{|l|}{ DII } \\
\hline Median & $-2 \cdot 6$ & -1.6 & -0.8 & 0.1 & 1.5 & & $-2 \cdot 7$ & -1.7 & -0.9 & -0.07 & $1 \cdot 3$ & \\
\hline Minimum, maximum & $-4 \cdot 9,-2$ & $-2,-1 \cdot 2$ & $-1 \cdot 2,-0.3$ & $-0.3,0.7$ & $0.7,3.7$ & & $-5 \cdot 2,-2 \cdot 2$ & $-2 \cdot 2,-1 \cdot 4$ & $-1.4,-0.5$ & $-0.5,0,5$ & $0.5,3.7$ & \\
\hline$n$ & 829 & 829 & 829 & 829 & 829 & & 619 & 618 & 618 & 618 & 618 & \\
\hline Age (years) & & & & & & $<0.001$ & & & & & & 0.180 \\
\hline Mean & 67 & 67 & 68 & 68 & 69 & & 66 & 66 & 66 & 66 & 66 & \\
\hline SD & 6 & 6 & 6 & 6 & 6 & & 6 & 6 & 7 & 7 & 7 & \\
\hline Family history of early $\mathrm{CHD}(\%)$ & 27 & 28 & 27 & 26 & 24 & 0.362 & 21 & 18 & 17 & 16 & 15 & 0.039 \\
\hline Hypertension (\%) & 84 & 86 & 87 & 87 & 87 & 0.359 & 76 & 78 & 79 & 79 & 77 & 0.436 \\
\hline Dyslipidaemia (\%) & 79 & 79 & 76 & 75 & 74 & 0.016 & 71 & 66 & 66 & 65 & 63 & 0.033 \\
\hline Diabetes (\%) & 44 & 40 & 44 & 44 & 49 & 0.011 & 53 & 53 & 53 & 56 & 56 & 0.625 \\
\hline Smoking (\%) & & & & & & 0.250 & & & & & & 0.176 \\
\hline Current smoker & 4 & 5 & 6 & 6 & 6 & & 24 & 24 & 25 & 28 & 27 & \\
\hline Former smoker & 7 & 8 & 8 & 8 & 6 & & 48 & 52 & 46 & 46 & 49 & \\
\hline Total energy intake (kcal/d) & & & & & & $<0.001$ & & & & & & $<0.001$ \\
\hline Mean & 2442 & 2281 & 2136 & 1990 & 1767 & & 2769 & 2577 & 2412 & 2252 & 2038 & \\
\hline $\mathrm{SD}$ & 478 & 449 & 435 & 414 & 400 & & 585 & 558 & 511 & 459 & 463 & \\
\hline \multicolumn{13}{|l|}{ Total energy intake $(\mathrm{kJ} / \mathrm{d})$} \\
\hline Mean & 10217 & 9544 & 8937 & 8326 & 7393 & & 11586 & 10782 & 10092 & 9422 & 8527 & \\
\hline SD & 2000 & 1879 & 1820 & 1732 & 1674 & & 2448 & 2335 & 2138 & 1920 & 1937 & \\
\hline Physical activity (MET-h/d) & & & & & & $<0.001$ & & & & & & $<0.001$ \\
\hline Mean & 3.4 & $2 \cdot 9$ & 3.0 & $2 \cdot 8$ & $2 \cdot 4$ & & $6 \cdot 1$ & $5 \cdot 3$ & $5 \cdot 1$ & $4 \cdot 8$ & 4.3 & \\
\hline SD & 3.2 & $2 \cdot 8$ & $2 \cdot 8$ & $2 \cdot 7$ & $2 \cdot 4$ & & $6 \cdot 0$ & 4.7 & 4.9 & 4.4 & 3.9 & \\
\hline Alcohol intake $(\mathrm{g} / \mathrm{d})$ & & & & & & 0.001 & & & & & & $<0.001$ \\
\hline Mean & $3 \cdot 8$ & 3.2 & $2 \cdot 7$ & $3 \cdot 3$ & $2 \cdot 7$ & & $18 \cdot 4$ & $15 \cdot 2$ & $15 \cdot 5$ & 14.5 & $13 \cdot 3$ & \\
\hline SD & $7 \cdot 6$ & $6 \cdot 1$ & $5 \cdot 1$ & $6 \cdot 1$ & $5 \cdot 7$ & & $18 \cdot 1$ & 17.5 & $19 \cdot 0$ & $18 \cdot 2$ & $17 \cdot 9$ & \\
\hline Marital status (\%) & & & & & & 0.004 & & & & & & 0.043 \\
\hline Married & 72 & 66 & 66 & 67 & 62 & & 92 & 91 & 89 & 89 & 86 & \\
\hline Widowed & 20 & 27 & 27 & 26 & 30 & & 3 & 3 & 3 & 3 & 6 & \\
\hline Single/other & 8 & 7 & 7 & 7 & 8 & & 5 & 6 & 8 & 8 & 8 & \\
\hline Educational level (\%) & & & & & & $<0.001$ & & & & & & 0.066 \\
\hline Primary education or less & 80 & 86 & 87 & 86 & 88 & & 64 & 66 & 71 & 69 & 67 & \\
\hline Secondary education & 13 & 11 & 9 & 11 & 9 & & 21 & 23 & 19 & 22 & 22 & \\
\hline Any college & 7 & 3 & 4 & 3 & 3 & & 15 & 12 & 11 & 10 & 11 & \\
\hline
\end{tabular}

$\mathrm{MET}$, metabolic equivalents.

*Highest anti-inflammatory values of the DI.

† Highest pro-inflammatory values of the DII. 
Table 2. Nutrient and food consumption according to quintiles (Q) of the dietary inflammatory index (DII) in the PREvención con Dleta MEDiterránea (PREDIMED) trial, 2003-9 (Mean values and standard deviations)

Quintiles of the DII

\begin{tabular}{|c|c|c|c|c|c|c|c|c|c|c|c|c|c|c|}
\hline & \multicolumn{7}{|c|}{ Women } & \multicolumn{7}{|c|}{ Men } \\
\hline & \multicolumn{2}{|c|}{ Q1* } & \multicolumn{2}{|c|}{ Q2-Q4 } & \multicolumn{2}{|c|}{ Q5† } & \multirow[b]{2}{*}{$P$} & \multicolumn{2}{|c|}{$\mathrm{Q} 1^{*}$} & \multicolumn{2}{|c|}{ Q2-Q4 } & \multicolumn{2}{|c|}{ Q5† } & \multirow[b]{2}{*}{$P$} \\
\hline & Mean & SD & Mean & SD & Mean & SD & & Mean & SD & Mean & SD & Mean & SD & \\
\hline Carbohydrate intake (\%E) & 43.4 & $7 \cdot 0$ & $42 \cdot 6$ & $6 \cdot 7$ & $40 \cdot 8$ & $6 \cdot 8$ & $<0.001$ & $41 \cdot 2$ & $7 \cdot 0$ & 41.5 & $7 \cdot 3$ & 39.2 & $7 \cdot 8$ & $<0.001$ \\
\hline Protein intake (\%E) & $17 \cdot 2$ & $2 \cdot 6$ & $17 \cdot 1$ & $2 \cdot 7$ & $17 \cdot 2$ & $3 \cdot 1$ & 0.452 & $16 \cdot 0$ & $2 \cdot 6$ & $15 \cdot 9$ & $2 \cdot 7$ & $16 \cdot 0$ & $2 \cdot 8$ & 0.542 \\
\hline Vegetal protein intake (\%E) & $6 \cdot 1$ & $1 \cdot 1$ & 5.4 & 1.0 & 4.9 & $1 \cdot 0$ & $<0.001$ & $5 \cdot 8$ & $1 \cdot 1$ & $5 \cdot 4$ & 1.0 & 4.9 & $1 \cdot 0$ & $<0.001$ \\
\hline Animal protein intake (\%E) & $11 \cdot 1$ & $2 \cdot 7$ & 11.6 & $2 \cdot 8$ & $12 \cdot 3$ & 3.2 & $<0.001$ & $10 \cdot 2$ & $2 \cdot 7$ & 10.5 & $2 \cdot 8$ & $11 \cdot 1$ & 2.9 & $<0.001$ \\
\hline Total fat intake (\%E) & 38.4 & $6 \cdot 8$ & 39.4 & $6 \cdot 6$ & 40.9 & 6.9 & $<0.001$ & 38.2 & 6.5 & 38.5 & $6 \cdot 7$ & 40.4 & $7 \cdot 2$ & $<0.001$ \\
\hline Saturated fat $(\% \mathrm{E})$ & $9 \cdot 2$ & $2 \cdot 0$ & $10 \cdot 0$ & $2 \cdot 1$ & $10 \cdot 8$ & $2 \cdot 4$ & $<0.001$ & $9 \cdot 3$ & $2 \cdot 1$ & 9.9 & $2 \cdot 2$ & $10 \cdot 8$ & $2 \cdot 4$ & $<0.001$ \\
\hline Monounsaturated fat (\%E) & $18 \cdot 2$ & 4.5 & $19 \cdot 7$ & 4.5 & $21 \cdot 0$ & 4.8 & $<0.001$ & $18 \cdot 3$ & $4 \cdot 2$ & $19 \cdot 2$ & 4.4 & $20 \cdot 9$ & 4.6 & $<0.001$ \\
\hline Polyunsaturated fat (\%E) & $7 \cdot 2$ & $2 \cdot 2$ & $6 \cdot 2$ & $2 \cdot 1$ & $5 \cdot 6$ & $1 \cdot 7$ & $<0.001$ & $7 \cdot 1$ & $2 \cdot 2$ & $6 \cdot 2$ & $2 \cdot 0$ & $5 \cdot 6$ & 1.6 & $<0.001$ \\
\hline Alcohol consumption (g/d) & 3.8 & 7.4 & $3 \cdot 0$ & $5 \cdot 8$ & $2 \cdot 8$ & $5 \cdot 8$ & 0.002 & 18.5 & $18 \cdot 1$ & $15 \cdot 0$ & $18 \cdot 2$ & $13 \cdot 4$ & $17 \cdot 8$ & $<0.001$ \\
\hline Fibre $(g / d)$ & 34.6 & $9 \cdot 3$ & 24.7 & $6 \cdot 1$ & $16 \cdot 1$ & 3.6 & $<0.001$ & 34.4 & 8.8 & $25 \cdot 8$ & 6.5 & $17 \cdot 1$ & 3.8 & $<0.001$ \\
\hline Vitamin $E(m g / d)$ & $12 \cdot 5$ & 3.7 & $9 \cdot 7$ & 3.7 & $7 \cdot 0$ & $2 \cdot 6$ & $<0.001$ & 13.4 & $4 \cdot 8$ & $10 \cdot 5$ & 4.0 & 7.5 & 2.5 & $<0.001$ \\
\hline Vitamin C (mg/d) & 289 & 99 & 201 & 70 & 123 & 42 & $<0.001$ & 271 & 98 & 195 & 72 & 122 & 44 & $<0.001$ \\
\hline Vitamin $A(\mu \mathrm{g} / \mathrm{d})$ & 1757 & 784 & 1235 & 640 & 847 & 485 & $<0.001$ & 1870 & 907 & 1325 & 1095 & 849 & 500 & $<0.001$ \\
\hline Vegetables $(\mathrm{g} / \mathrm{d})$ & 483 & 168 & 327 & 107 & 213 & 78 & $<0.001$ & 482 & 179 & 324 & 111 & 212 & 80 & $<0.001$ \\
\hline Fruits $(g / d)$ & 487 & 214 & 379 & 186 & 231 & 118 & $<0.001$ & 462 & 231 & 377 & 195 & 237 & 138 & $<0.001$ \\
\hline Cereals (g/d) & 143 & 78 & 132 & 76 & 111 & 63 & $<0.001$ & 175 & 96 & 165 & 95 & 138 & 86 & $<0.001$ \\
\hline Potatoes (g/d) & 96 & 58 & 80 & 48 & 62 & 39 & $<0.001$ & 102 & 58 & 86 & 46 & 70 & 40 & $<0.001$ \\
\hline Legumes $(g / d)$ & $25 \cdot 8$ & $16 \cdot 8$ & $19 \cdot 7$ & $12 \cdot 9$ & $14 \cdot 8$ & $7 \cdot 8$ & $<0.001$ & $26 \cdot 2$ & $15 \cdot 1$ & $21 \cdot 5$ & $14 \cdot 0$ & $16 \cdot 3$ & 8.4 & $<0.001$ \\
\hline Nuts $(g / d)$ & $18 \cdot 6$ & 18.4 & $8 \cdot 2$ & $10 \cdot 8$ & $3 \cdot 3$ & $5 \cdot 6$ & $<0.001$ & $20 \cdot 6$ & $19 \cdot 6$ & $10 \cdot 3$ & $12 \cdot 9$ & 4.9 & $8 \cdot 1$ & $<0.001$ \\
\hline Fish and seafoods $(\mathrm{g} / \mathrm{d})$ & 123.9 & $60 \cdot 6$ & $95 \cdot 2$ & $41 \cdot 7$ & $74 \cdot 8$ & $36 \cdot 5$ & $<0.001$ & $130 \cdot 7$ & $56 \cdot 2$ & $101 \cdot 0$ & 53.8 & 79.7 & $39 \cdot 3$ & $<0.001$ \\
\hline Meat and meat products $(\mathrm{g} / \mathrm{d})$ & $125 \cdot 0$ & $57 \cdot 3$ & $127 \cdot 2$ & $50 \cdot 9$ & 114.5 & $49 \cdot 1$ & $<0.001$ & 144.7 & $68 \cdot 8$ & $141 \cdot 1$ & $58 \cdot 3$ & $136 \cdot 0$ & 61.9 & 0.044 \\
\hline Dairy products $(g / d)$ & 435.5 & 238.4 & $408 \cdot 1$ & $221 \cdot 0$ & 381.4 & 218.9 & $<0.001$ & 369.1 & $222 \cdot 4$ & $342 \cdot 4$ & 204.7 & 319 & $205 \cdot 9$ & $<0.001$ \\
\hline Mediterranean diet score $(0-14)$ & 9.5 & 1.9 & 8.6 & 1.8 & $7 \cdot 7$ & 1.7 & $<0.001$ & 9.8 & 1.9 & 8.7 & 1.9 & 8.0 & 1.7 & $<0.001$ \\
\hline
\end{tabular}

$\%$ E, percentage of energy.

the DII.

thighest pro-inflammatory values of the DII. 
significant among women, except for the percentage of subjects with a family history of early CHD, the presence of hypertension and smoking status. Among men, differences between the quintiles of the DII according to age, hypertension, diabetes and smoking status were not statistically significant. In both sexes, the level of physical activity was inversely associated with the DII, as was total energy intake and alcohol intake.

All macro- and micronutrient intakes were higher in the quintile with the lowest DII score (anti-inflammatory dietary pattern), except for intakes of animal protein, saturated fat and monounsaturated fat, both among women and men (Table 2). Better adherence to a MeDiet also was associated with lower DII scores.

Table 3 shows the adjusted indices of obesity based on BMI, waist circumference and WHtR, according to the DII score stratified by sex. The lower and upper limits of this score are shown for each quintile. Mean values of all three adiposity indices increased linearly across the successive quintiles of DII scores (from anti-inflammatory to pro-inflammatory levels). A significant positive correlation was observed between these obesity indices and the DII score.

Among women, the DII was directly associated with BMI, after adjusting for multiple factors related to obesity (Table 4). Being in the highest quintile of the DII was associated with an increase in BMI of $0.79 \mathrm{~kg} / \mathrm{m}^{2}(95 \% \mathrm{CI} 0.35,1 \cdot 23)$ compared with the lowest quintile ( $P$ for trend $=0.001$ ). This association was not statistically significant for men.

Table 4 further shows that waist circumference and WHtR increased progressively across quintiles 2-4 and 5 compared with the lowest quintile of the DII, both in women and men ( $P$ for trend being statistically significant in all comparisons).

Table 5 shows the association of the DII with the anthropometric indices, after considering the possible contribution of the MeDiet elements to the anti- or pro-inflammatory capacity of the diet. A higher pro-inflammatory level of the diet (beyond the effect of lower adherence to the MeDiet) was associated with higher adjusted means of BMI, waist circumference and WHtR ( $P$ for trend $<0.05$ in all comparisons except for BMI among men). The predicted increase in anthropometric measures was statistically significant in women, except for the increase in BMI and WHtR, when the intermediate DII quintiles (2-4) were compared with the lowest category. On the contrary, the results were not statistically significant among men, except for waist circumference and WHtR, when comparing the highest $v$. the lowest quintile of the residuals of the DII.

\section{Discussion}

In the present study, we used the dietary inflammatory index (DII) score to assess the capacity of the overall dietary pattern to promote inflammation. Higher values of the DII represent a higher inflammatory potential of the diet. As expected, we observed that the DII was inversely associated with the intake of healthy foods, nutrients and adherence to the MeDiet. A pro-inflammatory DII was directly associated with the indices of general and abdominal obesity, independent

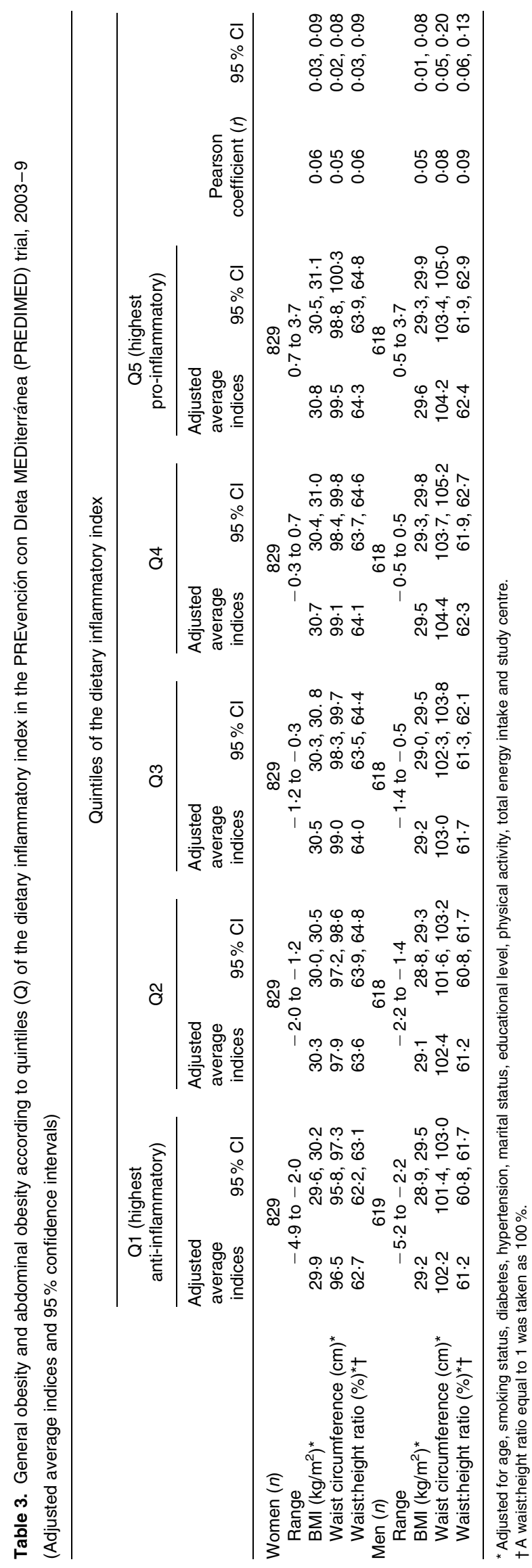


Table 4. Multivariable-adjusted ${ }^{*}$ differences in the indices of general obesity and abdominal obesity according to quintiles (Q) of the dietary inflammatory index in the PREvención con Dleta MEDiterránea (PREDIMED) trial, 2003-9

(Adjusted differences and $95 \%$ confidence intervals)

\begin{tabular}{|c|c|c|c|c|c|c|c|}
\hline & \multicolumn{7}{|c|}{ Quintiles of the dietary inflammatory index } \\
\hline & \multicolumn{2}{|c|}{$\begin{array}{l}\text { Q1 (highest anti-inflamma- } \\
\text { tory) }\end{array}$} & \multicolumn{2}{|c|}{ Q2-Q4 } & \multicolumn{2}{|c|}{ Q5 (highest pro-inflammatory) } & \multirow[b]{2}{*}{$P$ for trend } \\
\hline & $\begin{array}{l}\text { Adjusted } \\
\text { difference }\end{array}$ & $95 \% \mathrm{Cl}$ & $\begin{array}{l}\text { Adjusted } \\
\text { difference }\end{array}$ & $95 \% \mathrm{Cl}$ & $\begin{array}{l}\text { Adjusted } \\
\text { difference }\end{array}$ & $95 \% \mathrm{Cl}$ & \\
\hline \multicolumn{8}{|l|}{ Women } \\
\hline BMI $\left(\mathrm{kg} / \mathrm{m}^{2}\right)^{\star}$ & 0 & Reference & 0.56 & $0.23,0.89$ & 0.79 & $0.35,1.23$ & 0.001 \\
\hline Waist circumference $(\mathrm{cm})^{*}$ & 0 & Reference & 2.03 & $1 \cdot 17,2.90$ & 2.79 & $1.64,3.93$ & $<0.001$ \\
\hline Waist:height ratio $(\%)^{*} \dagger$ & 0 & Reference & $1 \cdot 19$ & $0.64,1.74$ & 1.60 & $0.87,2.33$ & $<0.001$ \\
\hline \multicolumn{8}{|l|}{ Men } \\
\hline BMI $\left(\mathrm{kg} / \mathrm{m}^{2}\right)^{\star}$ & 0 & Reference & 0.05 & $-0.26,0.37$ & 0.33 & $-0.08,0.74$ & 0.100 \\
\hline Waist circumference $(\mathrm{cm})^{*}$ & 0 & Reference & 0.98 & $0.07,1.89$ & 1.74 & $0.53,2.94$ & 0.005 \\
\hline Waist:height ratio $(\%)^{*} \dagger$ & 0 & Reference & 0.44 & $-0.09,0.97$ & 1.04 & $0.35,1.74$ & 0.003 \\
\hline
\end{tabular}

${ }^{*}$ Adjusted for age, smoking status, diabetes, hypertensive, physical activity, total energy intake, educational level, marital status and study centre.

† A waist:height ratio equal to 1 was taken as $100 \%$.

of established risk factors for obesity including total energy intake, age, smoking status, diabetes, hypertension, physical activity, educational level and marital status. These results were consistent for both sexes except for BMI in men. In the residual model (after removing the variability explained by the MeDiet), the association between the inflammatory potential of the diet and higher adiposity indices remained apparent; however, there was a clear association between the DII and the abdominal indices of obesity for women compared with men.

The associations observed between nutrient intake or food consumption and the DII are consistent with previous research. Several studies have shown an inverse association between healthy diets and markers of inflammation, as well as a direct association with 'Western-like' dietary patterns $^{(8-14)}$. Specifically, a lower CRP concentration has been associated with a higher intake of fruits and vegetables ${ }^{(34-36)}$, legumes ${ }^{(37)}$, nuts ${ }^{(38)}$, and low-fat dairy consumption ${ }^{(39)}$. Previous studies have also observed associations of specific nutrients such as total dietary fibre intake ${ }^{(40)}$, moderate alcohol consumption ${ }^{(41)}$, and vitamin $\mathrm{E}$ and vitamin $\mathrm{C}$ intake $^{(42)}$ with lower levels of inflammation markers. On the contrary, animal protein seems to increase the inflammatory status of obese individuals ${ }^{(43)}$.

We also found that a higher consumption of dairy products and meat (or meat products) was less frequent in the highest DII quintile. A systematic review has found no impact of dairy product consumption on biomarkers of inflammation in overweight and obese adults ${ }^{(44)}$. However, only one out of eight trials included in this review defined inflammation as its primary outcome, and there were some methodological limitations in them such as insufficient statistical power ${ }^{(44)}$. Concerning the consumption of meat, a cross-sectional analysis of data from 3690 diabetes-free female participants found that a higher intake of meat protein was associated with higher plasma levels of inflammatory markers ${ }^{(45)}$. However, a cross-sectional study has shown that the association between red meat intake and inflammatory markers was no

Table 5. Multivariable-adjusted ${ }^{*}$ differences in the indices of general obesity and abdominal obesity according to adherence to the residuals of the dietary inflammatory index on the fourteen-item PREvención con Dleta MEDiterránea (PREDIMED) score of adherence to the Mediterranean diet in the PREDIMED trial 2003-9

(Adjusted differences and $95 \%$ confidence intervals)

\begin{tabular}{|c|c|c|c|c|c|c|c|}
\hline & \multicolumn{7}{|c|}{ Quintiles of the dietary inflammatory index (adjusted for adherence to the Mediterranean diet) } \\
\hline & \multicolumn{2}{|c|}{$\begin{array}{c}\text { Q1 (highest } \\
\text { anti-inflammatory) }\end{array}$} & \multicolumn{2}{|c|}{ Q2-Q4 } & \multicolumn{2}{|c|}{$\begin{array}{c}\text { Q5 (highest } \\
\text { pro-inflammatory) }\end{array}$} & \multirow[b]{2}{*}{$P$ for trend* } \\
\hline & $\begin{array}{l}\text { Adjusted } \\
\text { difference }\end{array}$ & $95 \% \mathrm{Cl}$ & $\begin{array}{l}\text { Adjusted } \\
\text { difference }\end{array}$ & $95 \% \mathrm{Cl}$ & $\begin{array}{l}\text { Adjusted } \\
\text { difference }\end{array}$ & $95 \% \mathrm{Cl}$ & \\
\hline \multicolumn{8}{|l|}{ Women } \\
\hline BMI $\left(\mathrm{kg} / \mathrm{m}^{2}\right)^{\star}$ & 0 & Reference & $0 \cdot 14$ & $-0.19,0.48$ & 0.36 & $-0.08,0.80$ & 0.041 \\
\hline Waist circumference $(\mathrm{cm})^{*}$ & 0 & Reference & 1.01 & $0 \cdot 14,2 \cdot 81$ & 1.67 & $0.52,2.82$ & $<0.001$ \\
\hline Waist:height ratio $(\%)^{*} \dagger$ & 0 & Reference & 0.34 & $-0.20,0.91$ & 0.76 & $0.04,1.49$ & 0.007 \\
\hline \multicolumn{8}{|l|}{ Men } \\
\hline BMI $\left(\mathrm{kg} / \mathrm{m}^{2}\right)^{\star}$ & 0 & Reference & 0.06 & $-0.26,0.36$ & 0.31 & $-0.10,0.73$ & 0.057 \\
\hline Waist circumference $(\mathrm{cm})^{*}$ & 0 & Reference & 0.77 & $-0.14,1.69$ & 1.46 & $0.24,2.68$ & 0.004 \\
\hline Waist:height ratio $(\%)^{*} \dagger$ & 0 & Reference & 0.44 & $-0.09,0.96$ & 0.96 & $0.26,1.67$ & 0.005 \\
\hline
\end{tabular}

${ }^{*}$ Adjusted for age, smoking status, diabetes status, hypertensive status, physical activity, energy intake, educational level, marital status and study centre.

† A waist:height ratio equal to 1 was taken as $100 \%$. 
longer observed after adjustment for $\mathrm{BMI}^{(46)}$. Therefore, it is suggested that the association between red meat intake and inflammation is probably mediated by obesity.

In the present study, a higher pro-inflammatory diet was observed in participants with higher BMI, waist circumference and WHtR. This result suggests the hypothesis that a dietinduced inflammation might contribute to increasing or maintaining obesity, especially abdominal obesity, in a population that is mostly overweight or obese. The origin of inflammation during obesity is not yet fully understood. It is acknowledged that inflammation is induced by adiposity ${ }^{(4,5)}$, but this relationship can be bidirectional (i.e. a pro-inflammatory diet can increase or maintain adiposity), thus creating a vicious cycle, because nutrient excess and some specific foods or nutrients also have been associated with inflammation ${ }^{(47)}$ The potential mechanisms underlying this association is the activation of pathogen-associated molecular patterns, such as Toll-like receptors and Nod-like receptors, which induce the activation of inflammatory markers in several tissues including the adipose tissue ${ }^{(48)}$. Moreover, dietary patterns (e.g. highfat/low-fibre or low-fat/high-fibre diet) and single specific nutrients (e.g. dietary fibre) appear to have important consequences in the gut microbiota, which is also involved in low-grade inflammation associated with obesity ${ }^{(49-52)}$.

The residuals of the DII (from a regression model on adherence to the MeDiet) were also associated with obesity indices. These residuals represent the information provided by the DII about the anti- or pro-inflammatory capacity of a diet, which could not be explained by adherence to the MeDiet. The most pro-inflammatory diet showed a stronger association with waist circumference than with other anthropometric indices, both among women and men. These results are in close agreement with previous findings, which showed that central adiposity-related indices are more strongly correlated with plasma pro-inflammatory markers than indices assessing total adiposity in healthy young adults ${ }^{(5)}$. Moreover, abdominal adiposity has been associated with elevated CRP levels, independent of BMI in older adults ${ }^{(53)}$. As a consequence, the present results reinforce the usefulness of the DII to assess the inflammatory properties of a diet, and the association between inflammation and central obesity indices.

The present results are also consistent with those of studies reporting a stronger association between CRP and BMI in women than in men ${ }^{(54,55)}$. This between-sex difference could be partially explained by a greater accumulation of subcutaneous fat in women than in men, and higher lean mass in men ${ }^{(55)}$. Sex differences in the metabolic activity of adipose tissue, as well as in the association between leptin and CRP, may also explain these differences ${ }^{(56,57)}$.

The strengths of the present study include the following: large sample size; use of a validated instrument to measure the inflammatory potential of the diet; adjustment for a large number of factors associated with obesity; detailed measures of obesity indices; validation of all assessment instruments including the MeDiet screener, the FFQ and the physical activity questionnaire. The present study also has its limitations, the main one being the cross-sectional nature of our analyses. It is, therefore, unclear whether obese individuals are more likely to choose pro-inflammatory diets, or if pro-inflammatory diets contribute to promoting or maintaining obesity. Both weight reduction and an overall healthy dietary pattern have the capacity to reduce inflammatory markers. Thus, the association between the DII and obesity indices remains to be confirmed in prospective analyses. Another limitation is that anthropometric measures are surrogate markers of abdominal obesity. Waist circumference and WHtR do not differentiate between visceral adipose tissue and subcutaneous abdominal adipose tissue ${ }^{(58)}$. Therefore, we cannot determine whether the DII is more strongly associated with visceral, subcutaneous or both types of abdominal fat mass. Finally, the DII is limited by the existing knowledge of the inflammatory factors involved in obesity. However, the DII has been found to be associated with the following factors: inflammatory cytokines including CRP and IL-6 ${ }^{(33,59,60)}$; glucose intolerance component of the metabolic syndrome ${ }^{(59)}$; odds of asthma and of reduced $\mathrm{FEV}_{1}$ (forced expiratory volume in $1 \mathrm{~s}$ ) in an Australian population ${ }^{(60)}$. It has also been reportedly associated with a higher risk of colorectal cancer $^{(61)}$, prostate cancer ${ }^{(62)}$ and pancreatic cancer ${ }^{(63)}$.

In conclusion, the present findings indicate an association between anti-inflammatory values of the DII and intake of healthy foods and nutrients and higher adherence to the MeDiet. A pro-inflammatory diet is associated with elevated indices of central and abdominal obesity. This association suggests that the DII may have the capacity to help elucidate the role that diet plays in the development of obesity through inflammatory processes.

\section{Supplementary material}

To view supplementary material for this article, please visit http://dx.doi.org/10.1017/S0007114514004401

\section{Acknowledgements}

The authors are very grateful to all the participants of the study for their enthusiastic collaboration; the PREDIMED personnel for their excellent assistance; and the personnel of all affiliated primary care centres.

The PREDIMED trial was supported by the Instituto de Salud Carlos III (ISCIII), the official funding agency for Biomedical Research of the Spanish Government, through grants provided to research networks specifically developed for the trial: RTIC G03/140 (to R. E.), CIBERobn and RTIC RD 06/0045 (to M. A. M.-G.). J. R. H. was supported by an Established Investigator Award in Cancer Prevention and Control from the Cancer Training Branch of the National Cancer Institute (K05 CA136975). The authors also acknowledge grants received from Centro Nacional de Investigaciones Cardiovasculares CNIC 06/2007, Fondo de Investigación Sanitaria Fondo Europeo de Desarrollo Regional (PI04-2239, PI05/ 2584, CP06/00100, PI07/0240, PI07/1138, PI07/0954, PI07/ 0473, PI10/01407, PI11/01647 and PI1102505), Ministerio de Ciencia e Innovación (AGL-2009-13906-C02, AGL2010-22319C03), Fundación Mapfre 2010, Public Health Division of the Department of Health of the Autonomous Government of 
Catalonia and Generalitat Valenciana (ACOMP06109, GVACOMP2010-181, GVACOMP2011-151, CS2010-AP-111 and CS2011-AP-042), and a joint contract (CES09/030) with the ISCIII and the Health Department of the Catalan Government (Generalitat de Catalunya). The roles of the sponsors are as follows: the supplemental foods used in the study were generously donated by Patrimonio Comunal Olivarero and Hojiblanca from Spain (extra-virgin olive oil); the California Walnut Commission from Sacramento, CA (walnuts); and Borges S.A. (almonds) and La Morella Nuts (hazelnuts), both from Reus, Spain. CIBERobn and RTIC RD 06/0045 are initiatives of ISCIII, Spain. However, the sponsors played no role in the design, collection, analysis or interpretation of the data or in the decision to submit the manuscript for publication.

The authors' contributions are as follows: M. R.-C. and M. A. M.-G. contributed to the study concept and design; N. S., J. R. H., A. S.-T., D. C., J. S.-S., M. Fitó, J. R., J. F.-C., M. Fiol, J. M. S.-L., L. S.-M., X. P., R. E. and M. A. M.-G. contributed to the acquisition of the data; M. R.-C., N. S. and M. A. M.-G. analysed and interpreted the data; M. R.-C. drafted the manuscript. All authors critically revised the manuscript for important intellectual content.

R. E. served on the board of and received lecture fees from the Research Foundation on Wine and Nutrition (FIVIN); served on the boards of the Beer and Health Foundation and the European Foundation for Alcohol Research (ERAB); received lecture fees from Cerveceros de España and SanofiAventis; and received grant support through his institution from Novartis. E. R. served on the board of and received travel support, as well as grant support through his institution from the California Walnut Commission; served on the board of the Flora Foundation (Unilever); served on the board of and received lecture fees from Roche; served on the board of and received grant support through his institution from Amgen; received consulting fees from Damm and Abbott Laboratories; received consulting fees and lecture fees, as well as grant support through his institution from Merck; received lecture fees from Aegerion, AstraZeneca, Danone, Pace and Rottapharm; received lecture fees and payment for the development of educational presentations, as well as grant support through his institution from Ferrer; received payment for the development of educational presentations from Recordati; and received grant support through his institution from Daiichi Sankyo, Feiraco, Karo Bio, Nutrexpa, Pfizer, Sanofi-Aventis, Synageva, Takeda and Unilever. J. S.-S. served on the board of and received grant support through his institution from the International Nut and Dried Fruit Council; received consulting fees from Danone; and received grant support through his institution from Eroski and Nestlé. J. R. H. owns a controlling interest in Connecting Health Innovations LLC (CHI), a company planning to license the right to his invention of the dietary inflammatory index (DII) from the University of South Carolina in order to develop computer and smart phone applications for patient counselling and dietary intervention in clinical settings. N. S. is an employee of CHI. The subject matter of this paper will not have any direct bearing on that work, nor has that activity exerted any influence on this project.
The authors declare that there are no conflicts of interest despite the various aforementioned activities, payments and grants received.

\section{References}

1. Ng M, Fleming T, Robinson M, et al. (2014) Global, regional, and national prevalence of overweight and obesity in children and adults during 1980-2013: a systematic analysis for the Global Burden of Disease Study 2013. Lancet 384, 766-781.

2. WHO (2013) Obesity overweight. Fact sheet no. 311.

3. Heber D (2010) An integrative view of obesity. Am J Clin Nutr 91, 280S-283S.

4. Gregor MF \& Hotamisligil GS (2011) Inflammatory mechanisms in obesity. Annu Rev Immunol 29, 415-445.

5. Hermsdorff HH, Zulet MA, Puchau B, et al. (2011) Central adiposity rather than total adiposity measurements are specifically involved in the inflammatory status from healthy young adults. Inflammation 34, 161-170.

6. Emerging Risk Factors Collaboration, Wormser D, Kaptoge S, et al. (2011) Separate and combined associations of bodymass index and abdominal adiposity with cardiovascular disease: collaborative analysis of 58 prospective studies. Lancet 377, 1085-1095.

7. Ashwell M, Gunn P \& Gibson S (2012) Waist-to-height ratio is a better screening tool than waist circumference and BMI for adult cardiometabolic risk factors: systematic review and meta-analysis. Obes Rev 13, 275-286.

8. Schulze MB, Hoffmann K, Manson JE, et al. (2005) Dietary pattern, inflammation, and incidence of type 2 diabetes in women. Am J Clin Nutr 82, 675-684 (quiz 714-715).

9. Calder PC, Ahluwalia N, Brouns F, et al. (2011) Dietary factors and low-grade inflammation in relation to overweight and obesity. Br J Nutr 106, Suppl. 3, S5-S78.

10. Gogebakan O, Kohl A, Osterhoff MA, et al. (2011) Effects of weight loss and long-term weight maintenance with diets varying in protein and glycemic index on cardiovascular risk factors: the diet, obesity, and genes (DiOGenes) study: a randomized, controlled trial. Circulation 124, 2829-2838.

11. Ahluwalia N, Andreeva VA, Kesse-Guyot E, et al. (2013) Dietary patterns, inflammation and the metabolic syndrome. Diabetes Metab 39, 99-110.

12. Barbaresko J, Koch M, Schulze MB, et al. (2013) Dietary pattern analysis and biomarkers of low-grade inflammation: a systematic literature review. Nutr Rev 71, 511-527.

13. Kuczmarski MF, Mason MA, Allegro D, et al. (2013) Diet quality is inversely associated with C-reactive protein levels in urban, low-income African-American and white adults. J Acad Nutr Diet 113, 1620-1631.

14. Nettleton JA, Steffen LM, Mayer-Davis EJ, et al. (2006) Dietary patterns are associated with biochemical markers of inflammation and endothelial activation in the multi-ethnic study of atherosclerosis (MESA). Am J Clin Nutr 83, 1369-1379.

15. Viscogliosi G, Cipriani E, Liguori ML, et al. (2013) Mediterranean dietary pattern adherence: associations with prediabetes, metabolic syndrome, and related microinflammation. Metab Syndr Relat Disord 11, 210-216.

16. Salas-Salvadó J, Garcia-Arellano A, Estruch R, et al. (2008) Components of the Mediterranean-type food pattern and serum inflammatory markers among patients at high risk for cardiovascular disease. Eur J Clin Nutr 62, 651-659.

17. Urpi-Sarda M, Casas R, Chiva-Blanch G, et al. (2012) The Mediterranean diet pattern and its main components 
are associated with lower plasma concentrations of tumor necrosis factor receptor 60 in patients at high risk for cardiovascular disease. J Nutr 142, 1019-1025.

18. Babio N, Bulló M \& Salas-Salvadó J (2009) Mediterranean diet and metabolic syndrome: the evidence. Public Health Nutr 12, 1607-1617.

19. Schwingshackl L \& Hoffmann G (2014) Mediterranean dietary pattern, inflammation and endothelial function: a systematic review and meta-analysis of intervention trials. Nutr Metab Cardiovasc Dis 24, 929-939.

20. Bulló M, Casas-Agustench P, Amigo-Correig P, et al. (2007) Inflammation, obesity and comorbidities: the role of diet. Public Health Nutr 10, 1164-1172.

21. Funtikova AN, Benítez-Arciniega AA, Gomez SF, et al. (2014) Mediterranean diet impact on changes in abdominal fat and 10-year incidence of abdominal obesity in a Spanish population. Br J Nutr 111, 1481-1487.

22. Richard C, Couture P, Desroches S, et al. (2013) Effect of the Mediterranean diet with and without weight loss on markers of inflammation in men with metabolic syndrome. Obesity (Silver Spring) 21, 51-57.

23. Moreno-Aliaga MJ, Campion J, Milagro FI, et al. (2005) Adiposity and proinflammatory state: the chicken or the egg. Adipocytes 1, 1-16.

24. Marcason W (2010) What is the anti-inflammatory diet? J Am Diet Assoc 110, 1780

25. Shivappa N, Steck SE, Hurley TG, et al. (2014) Designing and developing a literature-derived, population-based dietary inflammatory index. Public Health Nutr 17, 1689-1696.

26. Estruch R, Ros E, Salas-Salvadó J, et al. (2013) Primary prevention of cardiovascular disease with a Mediterranean diet. $N$ Engl J Med 368, 1279-1290.

27. Martínez-Gonzalez MA, Corella D, Salas-Salvadó J, et al. (2012) Cohort profile: design and methods of the PREDIMED study. Int I Epidemiol 41, 377-385.

28. Martin-Moreno JM, Boyle P, Gorgojo L, et al. (1993) Development and validation of a food frequency questionnaire in Spain. Int J Epidemiol 22, 512-519.

29. Mataix J (2003) Tablas de Composición de Alimentos (Spanish Food Composition Tables), 4th ed. Granada: University of Granada.

30. Elosua R, Garcia M, Aguilar A, et al. (2000) Validation of the Minnesota leisure time physical activity questionnaire in Spanish women. Investigators of the MARATDON group. Med Sci Sports Exerc 32, 1431-1437.

31. Schroder H, Fito M, Estruch R, et al. (2011) A short screener is valid for assessing Mediterranean diet adherence among older Spanish men and women. J Nutr 141, 1140-1145.

32. Willett W (2013) Nutritional Epidemiology, 3rd ed. New York, NY: Oxford University Press.

33. Shivappa N, Steck SE, Hurley TG, et al. (2014) A populationbased dietary inflammatory index predicts levels of C-reactive protein in the seasonal variation of blood cholesterol study (SEASONS). Public Health Nutr 17, 1825-1833.

34. Esmaillzadeh A, Kimiagar M, Mehrabi Y, et al. (2006) Fruit and vegetable intakes, C-reactive protein, and the metabolic syndrome. Am J Clin Nutr 84, 1489-1497.

35. Root MM, McGinn MC, Nieman DC, et al. (2012) Combined fruit and vegetable intake is correlated with improved inflammatory and oxidant status from a cross-sectional study in a community setting. Nutrients $\mathbf{4}, 29-41$.

36. Hermsdorff HH, Zulet MA, Puchau B, et al. (2010) Fruit and vegetable consumption and proinflammatory gene expression from peripheral blood mononuclear cells in young adults: a translational study. Nutr Metab (Lond) 7, 42.
37. Hermsdorff HH, Zulet MA, Abete I, et al. (2011) A legumebased hypocaloric diet reduces proinflammatory status and improves metabolic features in overweight/obese subjects. Eur J Nutr 50, 61-69.

38. Casas-Agustench P, Lopez-Uriarte P, Bulló M, et al. (2011) Effects of one serving of mixed nuts on serum lipids, insulin resistance and inflammatory markers in patients with the metabolic syndrome. Nutr Metab Cardiovasc Dis 21, 126-135.

39. Esmaillzadeh A \& Azadbakht L (2010) Dairy consumption and circulating levels of inflammatory markers among Iranian women. Public Health Nutr 13, 1395-1402.

40. Ma Y, Griffith JA, Chasan-Taber L, et al. (2006) Association between dietary fiber and serum C-reactive protein. $A m J$ Clin Nutr 83, 760-766.

41. Imhof A, Froehlich M, Brenner H, et al. (2001) Effect of alcohol consumption on systemic markers of inflammation. Lancet 357, 763-767.

42. de Oliveira Otto MC, Alonso A, Lee DH, et al. (2011) Dietary micronutrient intakes are associated with markers of inflammation but not with markers of subclinical atherosclerosis. J Nutr 141, 1508-1515.

43. López-Legarrea P, de la Iglesia R, Abete I, et al. (2014) The protein type within a hypocaloric diet affects obesity-related inflammation: The RESMENA project. Nutrition 30, 424-429.

44. Labonte ME, Couture P, Richard C, et al. (2013) Impact of dairy products on biomarkers of inflammation: a systematic review of randomized controlled nutritional intervention studies in overweight and obese adults. Am J Clin Nutr 97, 706-717.

45. Ley SH, Sun Q, Willett WC, et al. (2014) Associations between red meat intake and biomarkers of inflammation and glucose metabolism in women. Am J Clin Nutr 99, $352-360$.

46. Montonen J, Boeing H, Fritsche A, et al. (2013) Consumption of red meat and whole-grain bread in relation to biomarkers of obesity, inflammation, glucose metabolism and oxidative stress. Eur J Nutr 52, 337-345.

47. Jin C \& Flavell RA (2013) Innate sensors of pathogen and stress: linking inflammation to obesity. J Allergy Clin Immunol 132, 287-294.

48. Salas-Salvadó J, Bulló M, Garcia-Lorda P, et al. (2006) Subcutaneous adipose tissue cytokine production is not responsible for the restoration of systemic inflammation markers during weight loss. Int J Obes (Lond) 30, 1714-1720.

49. Wu GD, Chen J, Hoffmann C, et al. (2011) Linking long-term dietary patterns with gut microbial enterotypes. Science 334, 105-108.

50. Martínez JA, Etxeberría U, Galar A, et al. (2013) Role of dietary polyphenols and inflammatory processes on disease progression mediated by the gut microbiota. Rejuvenation Res 16, 435-437.

51. Verdam FJ, Fuentes S, de Jonge C, et al. (2013) Human intestinal microbiota composition is associated with local and systemic inflammation in obesity. Obesity (Silver Spring) 21, E607-E615.

52. Chassaing B \& Gewirtz AT (2014) Gut microbiota, low-grade inflammation, and metabolic syndrome. Toxicol Pathol $\mathbf{4 2}$, 49-53.

53. Ahmadi-Abhari S, Luben RN, Wareham NJ, et al. (2013) Distribution and determinants of C-reactive protein in the older adult population: European Prospective Investigation into Cancer-Norfolk study. Eur J Clin Invest 43, 899-911.

54. Thorand B, Baumert J, Doring A, et al. (2006) Sex differences in the relation of body composition to markers of inflammation. Atherosclerosis 184, 216-224. 
55. Choi J, Joseph L \& Pilote L (2013) Obesity and C-reactive protein in various populations: a systematic review and meta-analysis. Obes Rev 14, 232-244.

56. Cartier A, Côté M, Lemieux I, et al. (2009) Sex differences in inflammatory markers: what is the contribution of visceral adiposity? Am J Clin Nutr 89, 1307-1314.

57. Rossi IA, Bochud M, Bovet P, et al. (2012) Sex difference and the role of leptin in the association between high-sensitivity C-reactive protein and adiposity in two different populations. Eur J Epidemiol 27, 379-384.

58. Klein S, Allison DB, Heymsfield SB, et al. (2007) Waist circumference and cardiometabolic risk: a consensus statement from shaping America's health: association for weight management and obesity prevention; NAASO, the obesity society; the American Society for Nutrition; and the American Diabetes Association. Am J Clin Nutr 85, $1197-1202$.
59. Cavicchia PP, Steck SE, Hurley TG, et al. (2009) A new dietary inflammatory index predicts interval changes in serum highsensitivity C-reactive protein. J Nutr 139, 2365-2372.

60. Wood LG, Shivappa N, Berthon BS, et al. (2015) Dietary inflammatory index is related to asthma risk, lung function and systemic inflammation in asthma. Clin Exp Allergy 45, $177-183$

61. Shivappa N, Prizment AE, Blair CK, et al. (2015) Dietary inflammatory index (DII) and risk of colorectal cancer in Iowa Women's Health Study. Cancer Epidemiol Biomarkers Prev 23, 2383-2392.

62. Shivappa N, Bosetti C, Zucchetto A, et al. (2015) Association between dietary inflammatory index and prostate cancer among Italian men. Br J Nutr 113, 278-283.

63. Shivappa N, Bosetti C, Zucchetto A, et al. (2015) Dietary inflammatory index and risk of pancreatic cancer in an Italian case-control study. Br J Nutr 113, 292-298. 